Results 114 bacteremic episodes in 83 patients were found. Incidence rate: $2.7 / 1,000$ patient-years (total $n: 3658$ ). At the time of the bacteremia: median age: 40.5 (8Đ90)years, 88.6\%female, disease duration:9.7 (IR16.7), medianSELENASLEDAI:4 (IR8), severe flare (SFI criteria):66\%, active nephritis:16.7\%, median SLICC/ACR DI: 3 (IR4), any comorbidity:64\% (McCabe-Jackson criteria: $28.1 \%$ rapidly/ultimately fatal), more frequently renal failure $(15.8 \%)$ or diabetes $(11.4 \%)$. SLE treatment at bacteremia: $88.6 \%$ corticosteroids $(68,6 \%>10 \mathrm{mg} /$ day $), 57 \%$ immunosuppresors (mycophenolate $17.5 \%$ and cyclophosphamide (CYP)12.3\%), 27\%antimalarials. $44.7 \%$ suffered invasive procedures, more frequently intravascular catheter (24.6\%). Nosocomial bacteremia in $35.1 \%$, more frequently urinary source (27.2\%). 64\% developed systemic inflammatory response syndrome, $35 \%$ needed intensive care unit admission, $22.8 \%$ had multiorganic failure. The most frequent microorganisms were E.coli (29.8\%), Staphylococcus aureus (16.7\%) (22\% methicillin-resistant) and Salmonella spp. (10.5\%). $16 \%$ of the gram-negative enteric bacilli were extendedspectrum b-lactamase positive. 17.5\%were multidrug resistant. $68.4 \%$ started the antibiotherapy before blood culture results, resulting finally active in susceptibility testing in 56 (71.8\%), indicating an appropriate empirical antibiotic therapy in 49\%. The bacteremia-related mortality was $14 \%$. Risk of death was higher in patients with severe sepsis (Pitt index >8) (OR: 13, 95\% CI: 3.71 to 45.17$)$. Bacteremia was recurrent in $26.3 \%$. Associations with bacteremia in bivariate analysis (114 bacteremias vs 688 controls) are shown in table 1 . Antimalarials were protective. In the multivariate analysis (adjusted for disease duration), only elevated creatinine (OR: 1.31 (95\% CI: 1.01 to 1.70$) \mathrm{p}=0.045)$, diabetes (OR $6.01(95 \%$ CI: 2.26 to 15.95$) \mathrm{p}=0.000)$, cancer (OR: $5.32(95 \%$ CI: 2.23 to 12.70$), \mathrm{p}=0.000$ ), immunosuppressors (OR: 6.35 (95\% CI: 3.42 to 11.77$) \mathrm{p}=0.000)$, CYP (OR: $9.37(95 \% \mathrm{CI}$ : 5.12 to 17.14$) \mathrm{p}=0.000)$ and SLICC/ACR DI (OR: 1.65 (95\% CI: 1.31 to 2.09$) \mathrm{p}=0.000)$ remained statistically significant.

Conclusion Bacteremia occurred mostly in active SLE, frequently in the context of a severe flare. Gram negative bacilli predominated, with high rate of multidrug resistance. The empiric treatment was inappropriate in a half of the cases. Recurrence and mortality were high. Immunosuppressors use, comorbidity and damage were all associated to bacteremia.

\section{S5D:6 SERIOUS INFECTION RATES IN SYSTEMIC LUPUS ERYTHEMATOSUS: A SWEDISH POPULATION-BASED ASSESSMENT}

1,2J Simard, 'M Rossides, ${ }^{1}$ E Arkema. ${ }^{1}$ Karolinska Institutet, Department of Medicine Solna, Stockholm, Sweden; ${ }^{2}$ Stanford School of Medicine, Department of Medicine, Department of Health Research and Policy, Stanford, USA

\subsection{6/lupus-2018-abstract.32}

Objective To investigate the rate of serious infections (SI) in systemic lupus erythematosus (SLE) compared to the general population.

Methods Individuals with incident SLE were identified from the Swedish National Patient Register (NPR) if they had two or more visits listing an SLE ICD code, at least one of which with a specialist, the first visit occurring Jan 2006 through Dec 2013. A non-SLE comparator group living in Sweden at the time the SLE case was diagnosed was matched on age, sex and county. We defined SI as a hospitalisation listing infection as main diagnosis from the NPR. Rates of SI per 1000 person-years (py) were calculated and age- and sexadjusted Cox models were used to estimate hazard ratios and 95\% confidence intervals ( $\mathrm{HR}, 95 \% \mathrm{CI}$ ) comparing SLE to non-SLE. Because individuals can experience more than one SI, we also calculated the rate of infection by year since SLE diagnosis including multiple infections.

Results We identified 2846 individuals with SLE and 13696 general population comparators. The average age at start of follow-up was $49 \%$ and $85 \%$ were female. Compared to the general population, individuals with SLE were more likely to have had a history of SI within a year before their first SLE visit $(7.8 \%$ vs $0.8 \%)$. The incidence rate for SI in SLE was

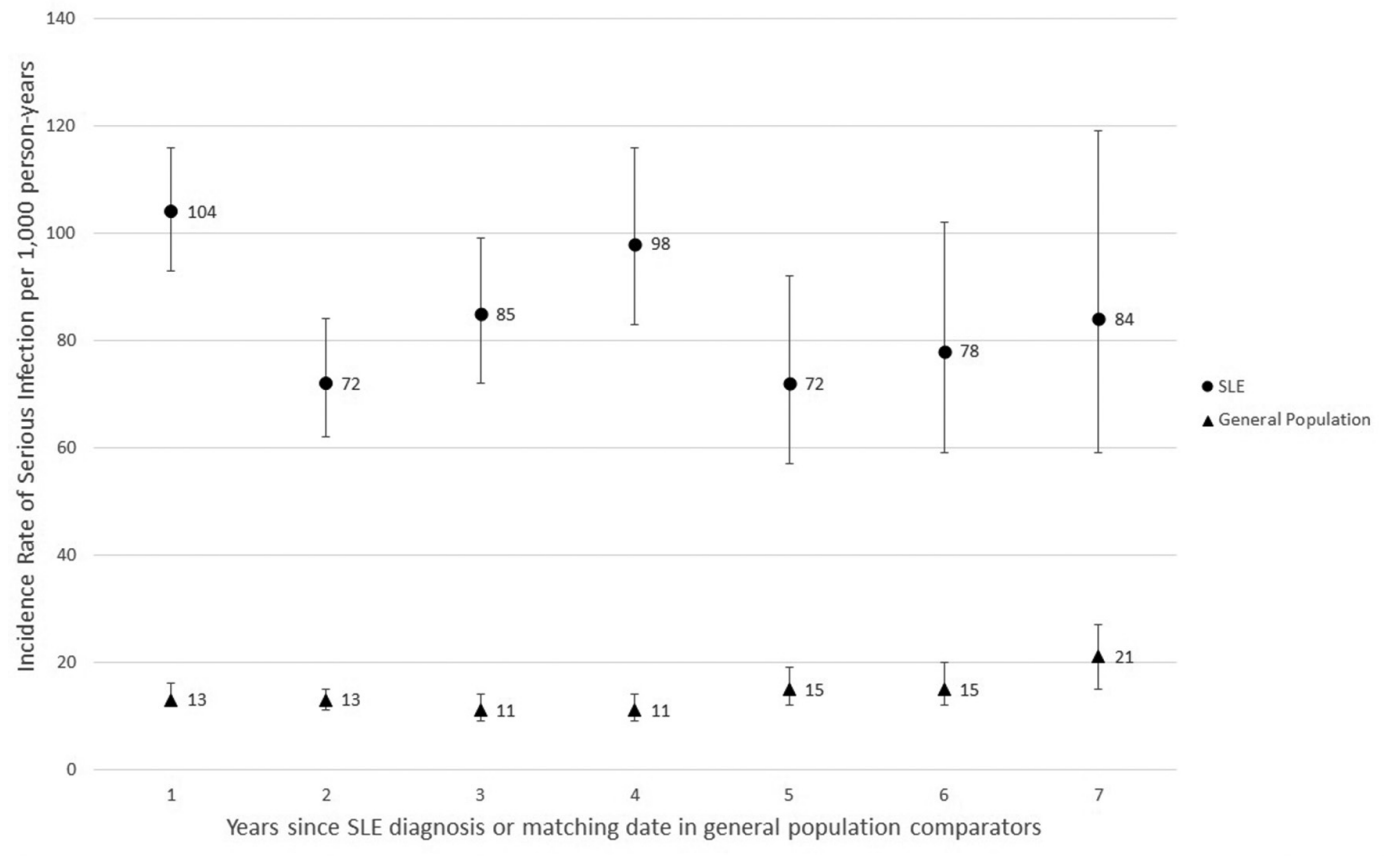

Abstract S5D:6 Figure 1 Incidence rates of serious infection per 1,000 person-year by time since diagnosis in systemic lupus erythematosus (SLE) and general population comparators 
$51 / 1000$ py, $26 \%$ of SIs were pneumonia and $1 \%$ were opportunistic infections. The HR for SI was 5.4 (95\% CI: 4.8 to 6.2) comparing SLE to non-SLE. Six percent of the SLE group experienced more than one SI over the study period. The rate of SI allowing for multiple events was 92/1000 py which varied over time since diagnosis (figure 1).

Conclusions The rate of SI in SLE is five times the rate in the general population, and remains high in the years following SLE diagnosis. The role of SLE treatments in infection risk should be investigated.

\section{S6a - Mantenaince Therapy}

\section{S6A:4 A POPULATION-BASED STUDY ON MORTALITY AND THE INFLUENCE OF MEDICATION USE IN 4356 PATIENTS WITH SYSTEMIC LUPUS ERYTHEMATOSUS AND 21845 MATCHED CONTROLS FROM THE UNITED KINGDOM}

${ }^{1} \mathrm{IE}$ Bultink, ${ }^{2,3} \mathrm{~F}$ de Vries, ${ }^{1} \mathrm{RF}$ van Vollenhoven, ${ }^{2,4} \mathrm{~A}$ Lalmohamed. ${ }^{1}$ Amsterdam Rheumatology and immunology Centre, location VUmc, Department of Rheumatology, Amsterdam, The Netherlands; ${ }^{2}$ Utrecht University, Department of Pharmacoepidemiology and Clinical Pharmacy, Utrecht, The Netherlands: ${ }^{3}$ Maastricht University Medical Centre, Department of Clinical Pharmacy and Toxicology, Maastricht, The Netherlands; ${ }^{4}$ University Medical Centre Utrecht, Department of Clinical Pharmacy, Utrecht, The Netherlands

\subsection{6/lupus-2018-abstract.33}

Purpose To estimate the magnitude of the risk from all-cause, age-specific, sex-specific, and cause-specific mortality in patients with SLE and relative risks compared with matched controls, and to evaluate the influence of medication exposure on mortality risk in SLE.

Methods We conducted a population-based cohort study using the Clinical Practice Research Datalink, Hospital Episode Statistics, and national death certificates (from 1987 to 2012). Each SLE patient $(n=4356)$ was matched with up to 6 controls $(n=21845)$ by age and sex. Multivariate Cox regression analysis estimated adjusted relative rates (RR) of mortality, and time interaction terms to evaluate mortality timing patterns. Time-dependent Cox models were used to evaluate the association of glucocorticoid use and hydroxychloroquine use on mortality and were adjusted for age, sex, lifestyle parameters, comorbidities and comedication.

Results A total of 442 out of 4356 SLE patients died during the study period. Patients with SLE had an increased mortality rate for all-cause mortality compared with age- and sexmatched subjects, after adjustment for confounders (adjusted RR 1.80, 95\% CI: 1.57 to 2.08). The RR was highest in patients aged 18-39 years (adjusted RR 4.87, 95\% CI: 1.93 to 12.3). Moratlity rates were not defferent between male and female patients. Glucocorticoid use in the previous six months raised the mortality rate while the adjusted RR was $45 \%$ decreased with low dose hydroxychloroquine use. SLE patients had increased cause-specific mortality rates for cardiovascular disease, infectious disease, noninfectious respiratory disease and for death due to accidents or suicide, while mortality rate for cancer was reduced, compared to age- and sex-matched subjects. The mortality rate was significantly increased for patients with a history of dementia, seizures, diabetes, cancer, and renal disease (table 1).

Conclusions Patients with SLE have a 1.8-fold increased mortality rate compared with the general population. Gluococorticoid use, female sex and young age are associated with an increased mortality risk while low dose hydroxychloroquine use significantly reduces the mortality rate. In addition, special attention should be paid to lupus patients with neuropsychiatric complications, diabetes, malignancy or renal disease since these subgroups of patients are at high risk of death.

\section{S6A:5 THE 3RS STRATEGY ONE YEAR LATER: STILL REACHING THE GOAL}

AV Taulaigo, A Lladó, M Vicente, MF Moraes-Fontes. Unidade de Doenças Auto-imunes, Hospital Curry Cabral/Serviço de Medicina 7.2, Centro Hospitalar Lisboa Central (CHLC) Lisbon, Portugal

10.1136/lupus-2018-abstract.34

Abstract S6A:4 Table 1 Risk of all-cause monthly within SLE patients $(n=4356)$, stratified according to organ damage (reference $=$ no risk factor)

\begin{tabular}{|l|c|c|c|c|}
\hline & $\begin{array}{c}\text { Person years } \\
(\mathbf{x} 1000)\end{array}$ & Deaths & $\begin{array}{c}\text { IR } \\
(/ 1000)\end{array}$ & Adjusted RR* (95\% CI) \\
\hline Dementia & 0.1 & 14 & 140.0 & $2.99(1.74-5.14)$ \\
\hline Seizures & 1.4 & 37 & 26.4 & $2.33(1.66-3.28)$ \\
\hline Cerebrovascular event & 1.9 & 73 & 38.4 & $1.28(0.99-1.65)$ \\
\hline Renal disease & 2.0 & 86 & 43.0 & $1.40(1.09-1.78)$ \\
\hline Osteoporotic fracture & 5.1 & 110 & 21.6 & $1.06(0.85-1.32)$ \\
\hline Use of antidiabetics & 0.9 & 45 & 50.0 & $1.90(1.39-2.59)$ \\
\hline Malignancy & 2.0 & 95 & 47.5 & $1.90(1.50-2.40)$ \\
\hline
\end{tabular}

* Adjusted for: recent use of corticosteroids, recent use of antimalarials, and recent use of benzodiazepines 